\title{
Acanthamoeba culbertsoni
}

National Cancer Institute

\section{Source}

National Cancer Institute. Acanthamoeba culbertsoni. NCI Thesaurus. Code C119299.

A species of motile, unicellular eukaryotic amoebae within the family of

Acanthamoebidae. A. culbertsoni is placed in morphological group III with a 18s rRNA

gene sequence type of T10. Members of this group are characterized by the presence of thin, smooth ectocysts and round endocysts. This species is a human pathogen and may cause granulomatous amoebic encephalitis and amoebic keratitis. 